\title{
Birefringence of a Block Copolymer Solution in the Stress Relaxation Process
}

\author{
Kunihiro OSAKI, Eiichi TAKatori, Michio Kurata, \\ Hiroshi OHNUMA, ${ }^{*}$ and Tadao KoTAKA* \\ Institute for Chemical Research, Kyoto University, \\ Uji, Kyoto 611, Japan \\ *Department of Macromolecular Science, Osaka University, \\ Toyonaka, Osaka 560, Japan
}

(Received July 15, 1986)

\begin{abstract}
The birefringence, $\Delta n$, and the shear stress, $\sigma$, were measured after an instantaneous shear deformation for a $10.5 \%$ solution of triblock copolymer, poly(methyl methacrylate)-block-polystyrene-block-poly(methyl methacrylate), in polychlorinated biphenyl. Hereafter $M$ stands for methyl methacrylate and $S$ for styrene. The mole fraction of $S$ of the copolymer was 0.4 . Measurements were performed at various magnitudes of shear, $\gamma$, ranging from 0.4 to 3 and $\Delta n$ was measured with a light led perpendicularly to the shear plane. Under assumptions that the $\Delta n$ and $\sigma$ are sums of independent contributions from $\mathrm{M}$ and $\mathrm{S}$ blocks and that the stress-optical law holds good for the contribution from each block, the stresses, $\sigma_{\mathrm{M}}$ and $\sigma_{\mathrm{S}}$, attributable to the $\mathbf{M}$ and $\mathbf{S}$ blocks, respectively, were separately evaluated. The result at short times was consistent with the assumption that the chain is uniformly deformed on instantaneous deformation of the material. The ratio $\sigma_{\mathrm{S}} / \sigma$ increased with time. For small $\gamma$, the result was consistent with the tube model theory if the ratio $\sigma_{\mathrm{S}} / \sigma$ was regarded as the fraction of stress attributable to the central portion of chain. At large deformations, the increase with time of the ratio was much less than expected from the theory.
\end{abstract}

KEY WORDS Birefringence / Stress Relaxation / Nonlinear Viscoelasticity /

Block Copolymer / Poly(methyl methacrylate-styrene-methyl methac-

rylate) / Entanglement / Tube Model /

The measurement of flow birefringence is a powerful method in rheological studies of polymeric liquids. ${ }^{1}$ For homopolymer systems, the birefringence and the stress are connected through a relation called the stress-optical law. This states that the anisotropic component of the refractive index tensor, $\Delta n$, is proportional to that of the stress tensor, $\sigma$ :

$$
\Delta n=C \sigma
$$

Here $C$ is called the stress-optical coefficient. The relation was revealed to be valid in timedependent flows as well as in steady flows. ${ }^{2-4}$ According to the stress-optical law, one can use birefringence measurement in the place of stress measurement when the latter is difficult for various reasons. ${ }^{1}$

The stress-optical coefficient is proportional to the anisotropy of the Kuhn statistical link of the chain, namely, to the difference between the polarizability parallel to the link and that perpendicular to the link. Thus the stress-optical coefficient depends on the monomer species. In the case of a block copolymer, the degree of anisotropy is not uniform along the chain but depends on the position along the chain. The stress-optical law will not hold valid unless the contribution to the stress of each segment is uniform along the chain. This, in turn, implies that the de- 
viation from the stress-optical law may represent the non-uniform contribution to the stress from various parts of the polymer chain.

Let us take a block copolymer of an ABAtype. Suppose the anisotropy of polarizability of the A segment is zero. Then the stress will be the sum of the contributions from the $A$ blocks, $\sigma_{\mathrm{A}}$, and from the B block, $\sigma_{\mathrm{B}}$, while the birefringence will include only the contribution from the $\mathrm{B}$ block, $C_{\mathrm{B}} \sigma_{\mathrm{B}}$. Thus one may be able to estimate the contribution to the stress from the central part of a polymer chain. Here it is assumed that the different blocks are homogeneously mixed and microphase separation does not exist. We also assume that the form birefringence is negligible. This is valid for homopolymer concentrates. ${ }^{1}$ For more general case of $C_{\mathrm{A}} \neq 0$, one may write

$$
\begin{gathered}
\boldsymbol{\sigma}=\boldsymbol{\sigma}_{\mathrm{A}}+\boldsymbol{\sigma}_{\mathrm{B}} \\
\Delta \boldsymbol{n}=C_{\mathrm{A}} \sigma_{\mathrm{A}}+C_{\mathrm{B}} \sigma_{\mathrm{B}}
\end{gathered}
$$

One may assume as a first approximation that $C_{\mathrm{A}}$ and $C_{\mathrm{B}}$ are equal to the stress-optical coefficients of homopolymers of $\mathrm{A}$ and $\mathrm{B}$, respectively. The assumption is supported by the fact that the stress-optical coefficient for homopolymer solutions are affected very little with varying solvents. ${ }^{1}$ If we measure $\sigma$ and $\Delta \boldsymbol{n}$ for a block copolymer, we can solve eq 2 for $\sigma_{\mathrm{A}}$ and $\sigma_{\mathrm{B}}$ and estimate the contributions to

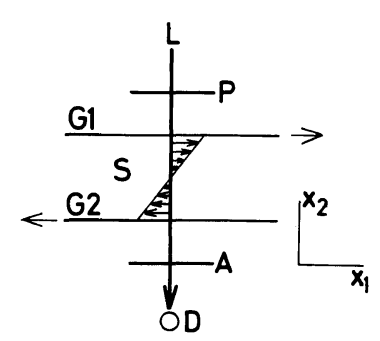

Figure 1. Shear deformation and prïnciple of birefringence measurement. Shear deformation is applied to test liquid, $\mathrm{S}$, by relative motion (thin arrows) of glass plates, G1 and G2. Light beam (thick arrow) is led through polarizer $P$, liquid $S$, and analyzer $A$, and its intensity is measured with optical detector, D. the stress from various parts of the chain.

As a start in applying the above-mentioned idea, we employ a simple birefringence measurement in a stepwise shear deformation (Figure 1):

$$
\begin{array}{ll}
u_{1}=u_{2}=u_{3}=0 & (t<0) \\
u_{1}=\gamma x_{2}, u_{2}=u_{3}=0 & (t \geqq 0)
\end{array}
$$

where $u_{j}$ represents the displacement in a Cartesian coordinate system $x_{1} x_{2} x_{3}$ and $\gamma$ is the magnitude of shear. We define a relaxation modulus at finite shear, $G(t, \gamma)$, from the shear stress, $\sigma$, on the $x_{3} x_{1}$ plane,

$$
G(t, \gamma)=\sigma / \gamma
$$

The limiting value at $\gamma \rightarrow 0$ of $G(t, \gamma)$ is the linear relaxation modulus, $G(t)$. We apply a light beam parallel to the $x_{2}$ axis and measure a birefringence, $\Delta n$. This corresponds to the difference of the principal values of an ellipse formed as the intersect of the refractive index ellipsoid and the shear plane. For homopolymer solutions, a simple approximate relation has been found good for the quantities $\Delta n$ and $\sigma .^{4-6}$

$$
\Delta n=C^{\prime} \sigma \gamma
$$

Here $C^{\prime}$ is a constant and is about $80 \%$ of the stress-optical coefficient, $C$. Thus, for this particular deformation history, one may replace the tensors in eq 2 with easily measurable components.

$$
\begin{gathered}
\sigma=\sigma_{\mathrm{A}}+\sigma_{\mathrm{B}} \\
\Delta n=C^{\prime}{ }_{\mathrm{A}} \gamma \sigma_{\mathrm{A}}+C^{\prime}{ }_{\mathrm{B}} \gamma \sigma_{\mathrm{B}}
\end{gathered}
$$

In the present study, we measure $\sigma$ and $\Delta n$ for a solution of a triblock copolymer, poly(methyl methacrylate)-block-polystyrene-block-poly(methyl methacrylate). The result will be compared with the chain deformation predicted by a model theory for entangled polymers.

\section{THEORETICAL}

First we take a polymer segment in a con- 
centrated solution or melt of a polymer. Suppose the segment is composed of $n$ Kuhn statistical links, with a polarizability $\alpha_{1}$ along its length and $\alpha_{2}$ perpendicular to its length. Then the increment of the stress and the polarizability tensors due to the segment are respectively given by ${ }^{7}$

$$
\begin{gathered}
\sigma=\frac{3 k T}{b^{2}}\langle\boldsymbol{r} \boldsymbol{r}\rangle \\
A=\frac{3}{5} \frac{\alpha_{1}-\alpha_{2}}{b^{2}}\langle\boldsymbol{r} \boldsymbol{r}\rangle
\end{gathered}
$$

where $\boldsymbol{r}$ is the end-to-end vector of the segment, $b^{2}$ is the average of $\mathbf{r}^{2}$ in free state, and $\langle\cdots\rangle$ implies the average in the pertinent state. Here we assumed that each segment contributes independently to the stress and polarizability. The assumption may be valid provided that the system is concentrated and the solution is uniform. The increment of stress and polarizability tensors are obtained as a sum of the quantities in eq 7 over all the segments in the volume. Provided that $\alpha_{1}-$ $\alpha_{2}$ is the same for all the segments, as is the case with a homopolymer, the increments of the stress and polarizability tensors are proportional to each other. The anisotropic component of the latter is proportional to the anisotropic component of the refractive index tensor if the former is small. Thus the stressoptical law follows. In the case of a block copolymer, the proportionality coefficients of $\langle\boldsymbol{r} \boldsymbol{r}\rangle$ in eq 7 depend on the monomer type. If one takes the summation for each type of segments separately, one obtains eq 6 .

\section{Tube Model}

We consider a tube model of Doi and Edwards $^{8}$ with $N$ tube segments per chain. Equation 7 holds good if the vector $\boldsymbol{r}$ is interpreted as the end-to-end vector of a tube segment. The 12-component of the tensor $\langle\boldsymbol{r r}\rangle$, which corresponds to the shear stress, in the stepwise shear deformation with small $\gamma$ is given by

$$
\begin{gathered}
\left\langle\boldsymbol{r}_{j} \boldsymbol{r}_{j}\right\rangle_{12}=\frac{\gamma b^{2}}{3} \sum_{p: \text { odd }}\left(\frac{4}{p \pi}\right) \sin \left(\frac{p \pi j}{N}\right) \exp \left(-\frac{t p^{2}}{\tau_{1}}\right) \\
(j=1,2,3, \cdots, N)
\end{gathered}
$$

Here $j$ indicates the tube-segment number counted from one end of the chain and $\tau_{1}$ is the reptation time characterizing the disengagement of the chain from the tube region defined at $t=0$. The summation should be taken over odd integers of $p$.

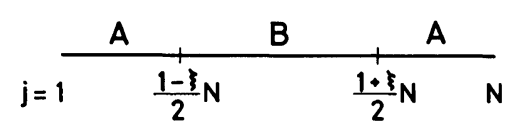

Figure 2. Triblock copolymer model. Tube-segment numbers for block boundaries are indicated.

Now we consider a block copolymer of an ABA type. We assume that all the properties of the units $A$ and $B$ but the anisotropy of polarizability are the same. The fractions of $\mathrm{A}$ and $\mathrm{B}$ units are $1-\xi$ and $\xi$, respectively, and the lengths of the two $A$ blocks are the same. Adding up the contribution from all the tube segments in unit volume, one can calculate the shear stress as

$$
\begin{aligned}
\sigma & =\frac{c N_{\mathrm{A}}}{M} \sum_{j=1}^{N} \frac{3 k T}{b^{2}}\left\langle\boldsymbol{r}_{j} \boldsymbol{r}_{j}\right\rangle_{12} \\
& =\frac{c R T}{M_{\mathrm{e}}} \sum_{j=1}^{N} \frac{3\left\langle\boldsymbol{r}_{j} \boldsymbol{r}_{j}\right\rangle_{12}}{N b^{2}} \\
& =\gamma \frac{c R T}{M_{\mathrm{e}}} \sum_{p: \text { odd }} \frac{8}{(p \pi)^{2}} \exp \left(-\frac{t p^{2}}{\tau_{1}}\right)
\end{aligned}
$$

Here $M$ is the molecular weight, $c$ is the mass concentration, $N_{\mathrm{A}}$ is the Avogadro number, $R$ is the gas constant and

$$
M_{\mathrm{e}}=\frac{M}{N}
$$

is the entanglement molecular weight or the molecular weight corresponding to a tube segment. The factor $c N_{\mathrm{A}} / M$ in the first line 


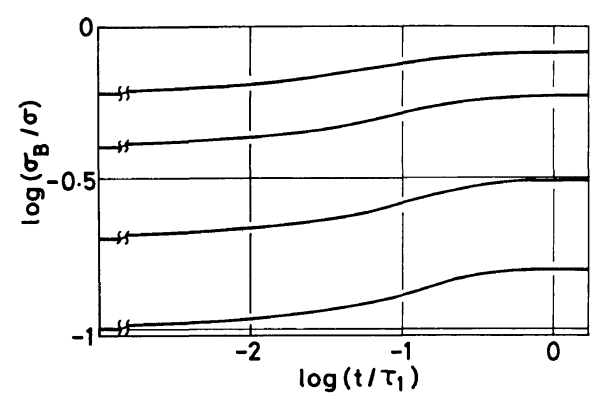

Figure 3. Ratio $\sigma_{\mathrm{B}} / \sigma$ plotted against reduced time $t / \tau_{1}$ for tube model. $\xi=0.6,0.4,0.2$, and 0.1 from top to bottom.

of eq 9 is the number of chains in unit volume and $\Sigma_{j}$ takes the sum over each chain.

For calculating the shear stress, $\sigma_{\mathrm{B}}$, originated by $B$ units, one has only to change the range of summation over $j$ in the second line of eq 9 .

$$
\begin{aligned}
\sigma_{\mathrm{B}} & =\frac{c R T}{M_{\mathrm{e}}} \sum_{j=N(1-\xi) / 2}^{N(1+\xi) / 2} \frac{3\left\langle\boldsymbol{r}_{j} \boldsymbol{r}_{\mathrm{j}}\right\rangle_{12}}{N b^{2}} \\
& =\gamma \frac{c R T}{M_{\mathrm{e}}} \sum_{p: \text { odd }} \frac{8}{(p \pi)^{2}} \exp \left(-\frac{t p^{2}}{\tau_{1}}\right) \cos \frac{p \pi(1-\xi)}{2}
\end{aligned}
$$

Evidently this reduces to $\sigma$ when $\xi=1$. The ratio $\sigma_{\mathbf{B}} / \sigma$ is plotted against a reduced time $t / \tau_{1}$ in Figure 3. For each value of $\xi$, the ratio increases with time, indicating the slower relaxation of the stress originated by the inner part of the chain. The limiting values of the ratio are

$$
\begin{aligned}
\sigma_{\mathrm{B}} / \sigma & =\xi \quad(t \rightarrow 0) \\
& =\sin \frac{\pi \xi}{2} \quad(t \rightarrow \infty)
\end{aligned}
$$

The value at the short time limit is equal to the fraction of $\mathrm{B}$ units in the molecule. This is the result of the assumed uniform deformation of the chain in the instantaneous deformation of the material. ${ }^{8}$

\section{Rouse Model}

One can perform similar calculations with the spring-beads model of Rouse, ${ }^{9}$ under the assumption that each bead displaces in proportion to the deformation of the material in the instantaneous deformation. The calculation is tedious but trivial and the result is

$$
\begin{gathered}
\sigma=\gamma \frac{c R T}{M} \sum_{p} \exp \left(-\frac{t p^{2}}{\tau_{1}}\right) \\
\sigma_{\mathbf{B}}=\gamma \frac{c R T}{M} \sum_{p} \exp \left(-\frac{t p^{2}}{\tau_{1}}\right)\left[\xi+\frac{\sin p \pi(1-\xi)}{p \pi}\right]
\end{gathered}
$$

where $\tau_{1}$ is the longest relaxation time. The summation is to be taken over positive integers of $p$. The limiting values of the ratio $\sigma_{\mathrm{B}} / \sigma$ are

$$
\begin{aligned}
\sigma_{\mathrm{B}} / \sigma & =\xi \quad(t \rightarrow 0) \\
& =\xi+\frac{\sin \pi \xi}{\pi} \quad(t \rightarrow \infty)
\end{aligned}
$$

Qualitative features of the ratio $\sigma_{\mathrm{B}} / \sigma$ are the same as those for the tube model. It may be noted that the long time limit of the ratio for the Rouse model is larger than that of the tube model at any value of $\xi$.

\section{MATERIAL AND METHOD}

The relaxation of stress and birefringence were measured for a solution of poly(methyl methacrylate)-block-polystyrene-blockpoly(methyl methacrylate) (PMSM for short) in polychlorinated biphenyl. The synthesis and characterization of the polymer, PMSM code 27B, were reported elsewhere. ${ }^{10}$ The number average molecular weight $\bar{M}_{n}$ of the styrene (S for short) block was $5.14 \times$ $10^{5}$ and the $\bar{M}_{n}$ of the copolymer was $1.24 \times$ $10^{6}$. The ratio of the weight-average molecular weight to $\bar{M}_{n}$ of the PMSM was 1.19 . The weight fraction of S as estimated from $\bar{M}_{n}$ is 0.415 and the mole fraction is 0.405 .

The solvent, Aroclor 1248, was a polychlorinated biphenyl supplied from Monsanto 
Chemical Company. Its viscosity and density were reported elsewhere. ${ }^{11}$ The refractive index of the solvent, $1.60,{ }^{12}$ is not much different from those of homopolymers corresponding to the components of PMSM; 1.60 for PS and 1.55 for PM (M stands for methyl methacrylate). ${ }^{13}$ This is another factor, in addition to the factor that the concentration is high, to make the effect of form birefringence small. ${ }^{13}$ The coefficients $C^{\prime}$ of eq 5 for the solutions in Aroclor of PS and PM are respectively,

$$
\begin{aligned}
& C^{\prime}{ }_{\mathrm{s}}=-5.0 \times 10^{-9} \mathrm{~Pa}^{-1} \\
& C^{\prime}{ }_{\mathrm{M}}=6.7 \times 10^{-10} \mathrm{~Pa}^{-1}
\end{aligned}
$$

These values are independent of the temperature and the magnitude of shear $\gamma$.

The solution was prepared with the procedure described earlier. ${ }^{11}$ The $20 \%$ solution of PMSM scattered light strongly and looked blue. The color faded on dilution. The $12 \%$ solution was clear and colorless to the naked eye. The measurements were performed for the $10.5 \%$ solution. Preliminary measurements of dynamic complex modulus revealed that the shape of viscoelastic functions and the temperature dependence were very similar to those of PM solutions with a comparable molecular weight. The straindependent relaxation moduli to be shown later are also similar to those of homopolymer solutions. Thus the polymer is likely to be dissolved homogeneously in this solution. These observations are in accord with those of Kitamura ${ }^{15}$ for solutions of a diblock copolymer, PMS, in polychlorinated biphenyl. The block copolymers of $S$ and M probably have little tendency of microphase separation in this solvent as suggested by him.

The details of the measurements of birefringence in the stress relaxation process were reported previously. ${ }^{5,6}$ Here the procedure is described roughly. The test liquid is filled in a gap between a pair of parallel glass plates as shown in Figure 1. The shear deformation is applied to the material by pulling the plates to opposite directions with a tensile tester. The shear stress, $\sigma$, is evaluated from the force applied to the plate. Two polarizing films, $\mathrm{P}$ and $\mathrm{A}$, are placed parallel to the glass plates. The angles between the direction of deformation and the polarizing directions of $P$ and $A$ are set equal to $\pi / 4$ and $-\pi / 4$, respectively. By measuring the intensity of light that passed through $\mathrm{P}$, test liquid, and $\mathrm{A}$, one can evaluate the birefringence, $\Delta n$, defined above eq 5 .

Measurements were performed at $20^{\circ} \mathrm{C}$ at various magnitudes of shear, $\gamma$, ranging from 0.4 to 3 .

\section{RESULTS AND DISCUSSION}

\section{Shear Stress and Birefringence}

The experimental results for the shear stress, $\sigma$, and the birefringence, $\Delta n$, are shown in Figrues 4 and 5, respectively: the straindependent relaxation modulus, $G(t, \gamma)=\sigma / \gamma$, in Figure 4 and the ratio $\Delta n / \gamma^{2}$ in Figure 5 . These quantities are independent of the magnitude of shear, $\gamma$, when $\gamma$ is small $(\leqq 0.7)$ and they decrease with increasing $\gamma$ at large $\gamma$. The curves at the top of Figures 4 and 5 represent the results at the limit of small $\gamma$.

At the limit of small $\gamma, G$ decays more rapidly with time than $\Delta n / \gamma^{2}$ does, especially

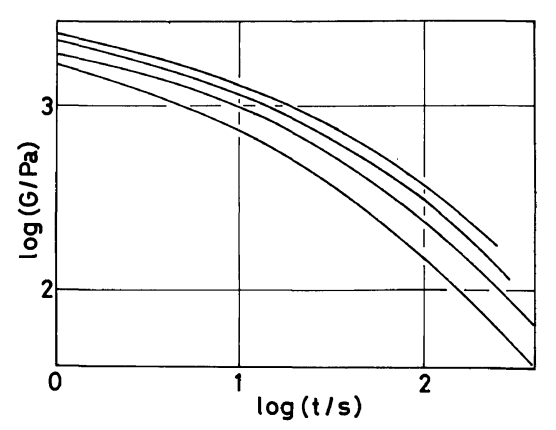

Figure 4. Strain-dependent relaxation modulus $G(t, \gamma)$ for PMSM solution. $\gamma=0,1.0,1.8$, and 3.0 from top to bottom. 


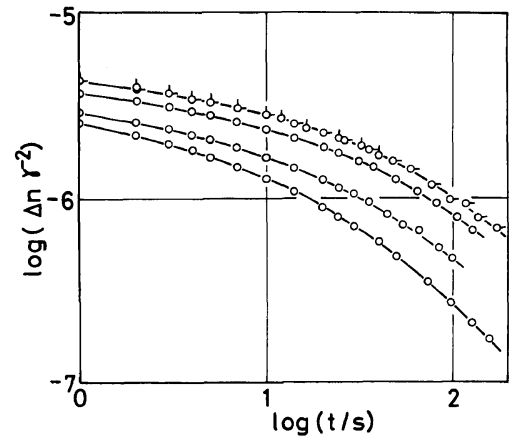

Figure 5. Ratio $\Delta n / \gamma^{2}$ for PMSM solution. $\gamma=0.36$ (pip up), 0.55 (pip right), 1.0, 1.8, and 3.0 from top to bottom.

at long times. As $\gamma$ increases, the relaxation rate increases for both of the quantities. At the highest $\gamma$ studied, the difference between the relaxation rates for $G$ and $\Delta n / \gamma^{2}$ is not very large. From a different view point one sees that the relative decreases of $G$ and $\Delta n / \gamma^{2}$ with increasing $\gamma$ is about the same at short times, while the decrease of $\Delta n / \gamma^{2}$ is much larger at long times: the nonlinearity at long times is more marked for $\Delta n / \gamma^{2}$ than for $G$.

Evidently eq 5 does not hold valid for this solution. We apply eq 6 to the data shown above and estimate the portions of stress attributable to the $\mathrm{M}$ and $\mathrm{S}$ blocks.

\section{Stress Attributable to $M$ and $S$ Blocks}

Equation 6 may be rewritten as

$$
\begin{gathered}
G=G_{\mathrm{M}}+G_{\mathrm{S}} \\
\frac{\Delta n}{\gamma^{2}}=C_{\mathrm{M}}^{\prime} G_{\mathrm{M}}+C_{\mathrm{S}}^{\prime} G_{\mathrm{S}}
\end{gathered}
$$

where the suffixes $A$ and $B$ are changed to $M$ and $S$, respectively, and the notations

$$
G_{\mathrm{M}}=\sigma_{\mathrm{M}} / \gamma, G_{\mathrm{S}}=\sigma_{\mathrm{S}} / \gamma
$$

were used. Equation 17 can be solved for $G_{\mathrm{M}}$ and $G_{\mathrm{S}}$ with the use of the data of Figures 4 and 5 and the coefficients given in eq 16 .

The results for the limit of small $\gamma$ and for $\gamma=3$ are shown in Figure 6. At short times, the

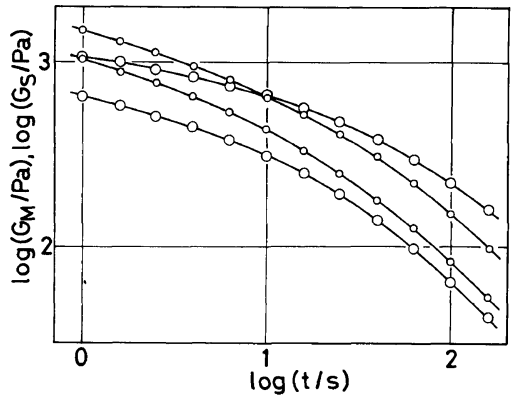

Figure 6. Relaxation moduli $G_{\mathrm{M}}$ (small circles) and $G_{\mathrm{S}}$ (large circles). $\gamma=0$ for upper pair and $\gamma=3$ for lower one.

quantity $G_{\mathrm{M}}$ (small circles) is larger than $G_{\mathrm{S}}$ (large circles). The former decreases more rapidly than the latter with time. At the limit of small deformation, $G_{\mathrm{S}}$ exceeds $G_{\mathrm{M}}$ at long times, $t>10 \mathrm{~s}$. At $\gamma=3$, the difference between the relaxation rates of $G_{\mathrm{M}}$ and $G_{\mathrm{S}}$ is not so large as at $\gamma=0$ and $G_{\mathrm{M}}$ remains larger than $G_{\mathrm{S}}$ over the whole range of time of measurement. The difference in the relaxation rates of $G_{\mathrm{M}}$ and $G_{\mathrm{S}}$ is as expected from the earlier model calculations for portions of stress supported by the segments located at different parts of the chain.

For more detailed study of the result, the ratio $G_{\mathrm{S}} / G$ is plotted against time in Figure 7 for the cases of $\gamma=0$ and $\gamma=3$. The ratio increases with time and decreases with magnitude of shear $\gamma$. At short times, the effect of varying $\gamma$ is small and the data points for various $\gamma$ seem to approach a common limiting value, about 0.4 , at a still shorter time. Actually one sees that the points for $\gamma=3$ tend to level off at short times. The limiting value seems to be approximately equal to the fraction of S units of the copolymer. This observation is consistent with a simple assumption that on instantaneous deformation of the material, the polymer chain is deformed uniformly in proportion to the material deformation. The contributions from segments located at different points along the chain would be the same at the instant of deformation. Incidentally, the assumption is incor- 


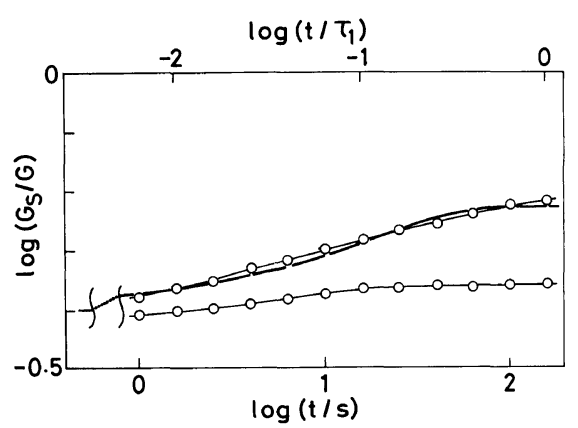

Figure 7. Ratio $G_{\mathrm{S}} / G$. Upper circles and thin line are experimental results for $\gamma=0$ and lower ones are for $\gamma=3$, plotted against $t$. Thick line is result of tube model theory plotted against $t / \tau_{1}$.

porated in model calculations shown in a previous section.

The thick solid line in Figure 7 represents the theoretical result of the tube model theory for the ratio $G_{\mathrm{S}} / G$ with $\xi=0.4$. The parameter $\tau_{1}$ is arbitrarily chosen so that the line fits the data for small $\gamma$. One may say that the theoretical result well represents the observed rate and amount of increase with time of the ratio $G_{\mathrm{S}} / G$ at small deformation. On the other hand, we do recognize that the shape of the relaxation curves in Figures 4 through 6 cannot be fitted with theoretical curves, which are very close to single exponential decay curves. More complete comparisons with the theory will be possible if the polymers with higher molecular weights and with sharper molecular weight distributions are available.

For a large deformation, $\gamma=3$, the ratio $G_{\mathrm{S}} / G$ does not increase so much and levels off at a much lower value than predicted by the theory. The solid line in Figure 7 is expected to be applicable also to large deformations at the present level of theory. ${ }^{8,16}$ The relevant assumptions in the current version of the theory may be that the stress relaxation proceeds only in two processes, the equilibration of the fluctuation of the chain contour length and the disengagement of the chain from a deformed tube, and that the former process is completed before the latter process becomes effective.
It would be interesting to calculate $\sigma_{\mathrm{B}}$ at large $\gamma$ with modified assumptions. ${ }^{16}$

\section{Nonlinearity at Long Times}

Finally we examine the degree of nonlinearity at long times. For homopolymer solutions, we define a function ${ }^{8,17}$

$$
h(\gamma)=\frac{G(t, \gamma)}{G(t)} \quad\left(t \gg \tau_{1}\right)
$$

This function was found to be a universal function for various polymeric systems, ${ }^{17}$ and its strain dependence was well described with the tube model theory; in short, it represents the decrease of stress due to the shrink of highly extended chains in the equilibration process of the fluctuation of chain contour length. ${ }^{8}$ We add an obvious extension of eq 18.

$$
h_{\mathrm{S}}(\gamma)=\frac{G_{\mathbf{S}}(t, \gamma)}{G_{\mathrm{S}}(t)}, h_{\mathrm{M}}(\gamma)=\frac{G_{\mathrm{M}}(t, \gamma)}{G_{\mathrm{M}}(t)} \quad\left(t \gg \tau_{1}\right)
$$

Unfortunately, data at sufficiently long times are not available. So we take the ratios defined in eq 18 and 19 at $t=100 \mathrm{~s}$ and show them in Figure 8. The curves represent theoretical results of tube model theory; the solid line, the result without a mathematical assumption called the independent alignment approximation (IAA) and the dashed line, the result with IAA.

The filled circles representing $h$ for the total shear stress is close to the dashed line. The result is very similar to that for homopolymer

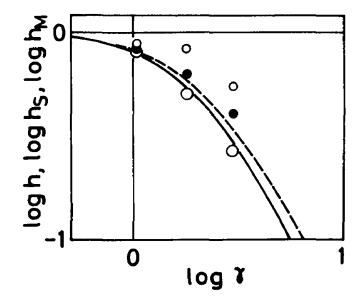

Figure 8. Nonlinearity functions $h$ (filled circles), $h_{\mathrm{M}}$ (small unfilled circles), and $h_{\mathrm{s}}$ (large unfilled circles), taken at $t=100 \mathrm{~s}$. Lines are prediction of tube model theory; solid line, without IAA; dashed line, with IAA. 
solutions. ${ }^{8,17}$ The large circles, for $h_{\mathrm{S}}$, representing the nonlinearity of the central part of the chain, lie lower and close to the solid line. This result can be favorably compared with the theory because the solid line is obtained without the surplus assumption of IAA on one hand, and the central part of chain may better be described with the tube model concept than the chain ends are. The result for $h_{\mathrm{M}}$ (small circles) indicates that the nonlinearity is very weak for the stress attributable to the segments located close to the chain ends. Such a behavior is not unexpected; the entanglement effect would be weaker for such segments and free polymer chains (not entangled) are not expected to exhibit much nonlinearity. The fact that $h$ for homopolymer solutions is always slightly higher than the theoretical value $^{8,17}$ may well be due to the weak nonlinearity of the stress attributable to the chain ends. Again it would be interesting to develop the theory modifying the assumptions of the idealized tube model as mentioned at the bottom of the last subsection.

Acknowledgement. This study was supported by the Grant-in-Aid for Scientific Research (No. 59550612, 1985) from the Ministry of Education, Science, Culture of Japan.

\section{REFERENCES}

1. See for example, H. Janeschitz-Kriegl, "Polymer Melt Rheology and Flow Birefringence," SpringerVerlag, Berlin, 1983.

2. F. H. Gortemaker, M. G. Hansen, B. de Cindio, H. M. Laun, and H. Janeschitz-Kriegl, Rheol. Acta, 15, 256 (1976).

3. K. Osaki, N. Bessho, T. Kojimoto, and M. Kurata, J. Rheol., 23, 457 (1979).

4. - K. Osaki, N. Bessho, T. Kojimoto, and M. Kurata, J. Rheol., 23, 617 (1979).

5. S. Kimura, K. Osaki, and M. Kurata, J. Polym. Sci., Polym. Phys. Ed., 19, 151 (1981).

6. S. Kimura, K. Osaki, and M. Kurata, J. Polym. Sci., Polym. Phys. Ed., 19, 517 (1981).

7. See Chapter 2 of ref 1.

8. M. Doi and S. F. Edwards, J. Chem. Soc., Faraday Trans. 2, 74, 1789, 1802 (1978).

9. P. E. Rouse, J. Chem. Phys., 21, 1272 (1953).

10. H. Ohnuma, T. Kotaka, and H. Inagaki, Polymer, 10, 501 (1969).

11. K. Osaki, Y. Nishimura, and M. Kurata, Macromolecules, 18, 1153 (1985).

12. N. Bessho, K. Osaki, and M. Kurata, Nihon Reoroji Gakkaishi, 5, 68 (1977).

13. V. N. Tsvetkov, in "Newer Methods of Polymer Characterization," B. Ke, Ed., Interscience Pub., New York, N. Y., 1964, Chapter 14.

14. K. Osaki, T. Takatori, M. Kurata, H. Watanabe, and T. Kotaka, to be published.

15. M. Kitamura, S. Yamamoto, T. Masuda, and S. Onogi, Nihon Reoroji Gakkaishi, 6, 20 (1978).

16. W. W. Graessley, Adv. Polymer Sci., 47, 67 (1982).

17. K. Osaki and M. Kurata, Macromolecules, 13, 671 (1980). 